\title{
A synthesis of 2,3-dihydro-4-pyridones
}

\author{
Michael Harmata* and Dong Reyoul Lee \\ Department of Chemistry, University of Missouri-Columbia Columbia, Missouri 65211, USA \\ E-mail: harmatam@missouri.edu
}

\section{Dedicated to Professor Lutz Tietze, a good friend and outstanding scientist, on the occasion of his $65^{\text {th }}$ birthday}

\begin{abstract}
The reaction of 1-alkoxy-1,4-dien-3-ones with primary amines results in the formation of dihydropyridones in good yield.
\end{abstract}

Keywords: Dihydropyridone, retro-Nazarov reaction, dienones

\section{Introduction}

Dihydropyridones have become extremely important intermediates in the synthesis of natural products, particularly alkaloids, and many approaches to their synthesis have been developed. ${ }^{1}$ However, the versatility of the ring system continues to spark interest in new synthetic developments aimed at establishing this ring system.

We recently reported the formation of 1-alkoxy-1,4-dien-3-ones via the retro-Nazarov reaction of substituted cyclopentanones (Scheme 1). ${ }^{2}$ This reaction is easy to perform and generally affords products in good- to excellent yields with essentially complete stereocontrol. Dienones such as those produced by this reaction have not been explored extensively with respect to their synthetic potential. It occurred to us that the reaction of these dienones with the appropriate nucleophile could result in ring formation. Our first efforts involved reactions with primary amines with the aim of executing two Michael addition reactions along with an elimination of an alkoxy group, to establish the dihydropyridone ring system. While this work was in progress, Dong and his co-workers reported a beautiful reaction of certain ketene thioacetals with primary amines to form dihydropyridones (Scheme 2 ). ${ }^{3}$ 


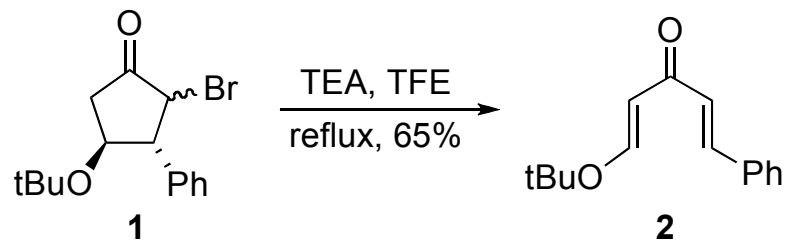

Scheme 1. An example of the retro-Nazarov reaction.<smiles>CCSc1[se]c(C#N)cc(=O)c1C(=O)OCc1ccccc1</smiles><smiles>CNC1=C(C(=O)c2ccccc2)C(=O)CC(c2ccccc2)N1C</smiles>

Scheme 2. Reaction of a ketene thioacetal with a primary amine. ${ }^{3}$

We initially tested the concept in a one-pot process. Thus, treatment of 5 with 1.1 equivalents of 2,2,6,6-tetramethylpiperidine in refluxing hexafluoro-isopropanol presumably afforded the retro-Nazarov product 6 (Scheme 3). This product was not isolated. Rather, 1 equivalent of benzylamine was added to the reaction mixture and the solution was heated at reflux for an additional 4 hours. This afforded a 50\% isolated yield of dihydropyridone $7 \mathbf{a}$ along with a $12 \%$ yield of the vinylogous amide $7 \mathbf{b}{ }^{4}$ It should be noted that attempts to cyclize $7 \mathbf{b}$ and various congeners to produce the corresponding pyridones were not successful. The implication is that attack of the benzylamine occurred initially on the C4-C5 double bond to afford 7. Attack at the double bond bearing the alkoxy group is a dead-end for formation of dihydropyridones. Although this procedure was interesting, we decided not to attempt optimization, but to explore the last step of the process with clean starting material in order to ascertain the efficiency of that process.

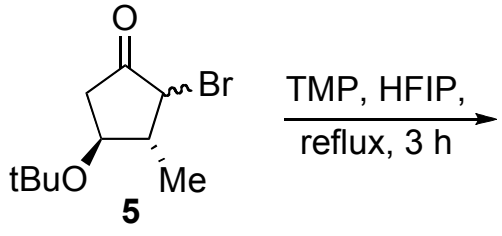

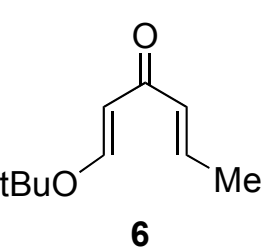

6

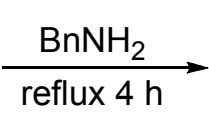

$\underbrace{O}_{\substack{1 \\ \text { Bn }}}$

$7 a, 50 \%$

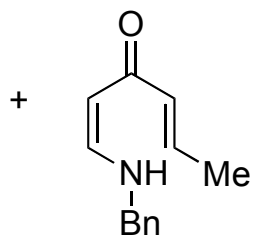

$7 b, 12 \%$

Scheme 3. One pot synthesis of a dihydropyridone.

We then conducted a brief exploration of the scope of the reaction. Since retro-Nazarov reactions are typically conducted in trifluoroethanol or HFIP, we used the former, less expensive, solvent to study the process. The reactions were conducted using stoichiometric amounts of the 
reactants as a $0.04 M$ solution in TFE at reflux for 12 hours. The results are summarized in Table 1. As shown in the Table, primary amines led to very high yields of dihydropyridones when 6 was used as a substrate (Table 1, entries 1-10). Interestingly, with 9 the yield of dihydropyridone was relatively low and $25 \%$ of the corresponding vinylogous amide $\mathbf{2 1 b}$ was isolated (Table 1, entry 12). This suggests a steric effect that either slows addition to the appropriate (C4-C5) double bond or increases the propensity for reversible addition at the same position. In either case, the double bond bearing the $t$-butoxy group should be attacked more frequently to afford a vinylogous amide rather than a dihydropyridone. Also of interest is the fact that the phenylsubstituted dienone $\mathbf{1 0}$ gave predominantly vinylogous amide $\mathbf{2 2 b}$ and only a small amount of the desired dihydropyridone 22a (Table 1, entry 13). It is hard to imagine this as a steric effect. It is possible that the loss of conjugation that occurs upon addition to the phenyl-substituted double bond increases reversibility such that starting material is drained away via the undesired reaction manifold. Many other dienones, amines, and reaction conditions need to be explored to understand all of the reactivity issues inherent in this process. ${ }^{`}$

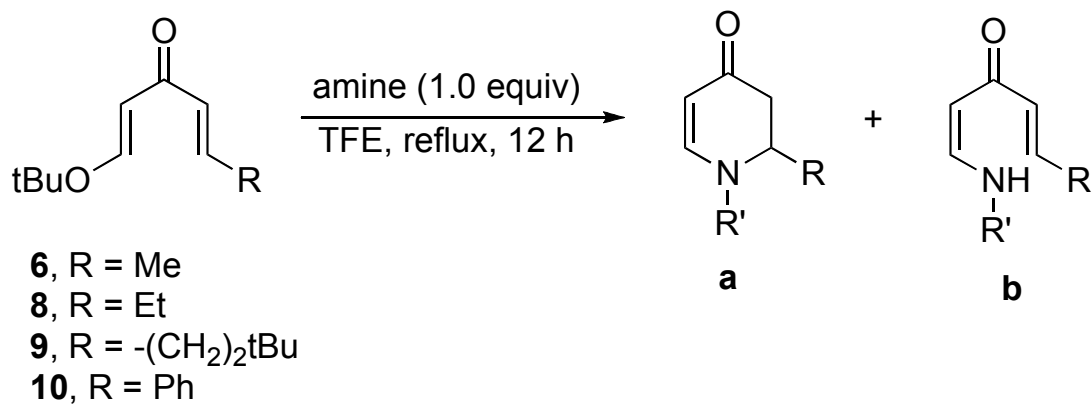

Pure TFE is not necessary for the success of the reactions we have discussed. Treatment of 6 with benzylamine in a 10:1 mixture of 1,2-dichloroethane and TFE at reflux for 4 hours gave a $92 \%$ yield of $7 \mathbf{a}$ and only a small amount of $7 \mathbf{b}$. We also examined the reaction of 6 with dibenzylamine. In this case, using standard reaction conditions, the only product isolated was the vinylogous amide 23 (Scheme 4). The stereochemical assignment of the product was based on the coupling constants between the olefinic hydrogens $(\mathrm{C} 1 / \mathrm{C} 2,12.7 \mathrm{~Hz}, \mathrm{C} 4 / \mathrm{C} 515.8 \mathrm{~Hz})$. Further examples of the reaction of secondary amines with dienones such as $\mathbf{6}$ must be performed to help us to understand the mechanistic basis of the course of the reactions, though we assume at present that it is subject to thermodynamic control.
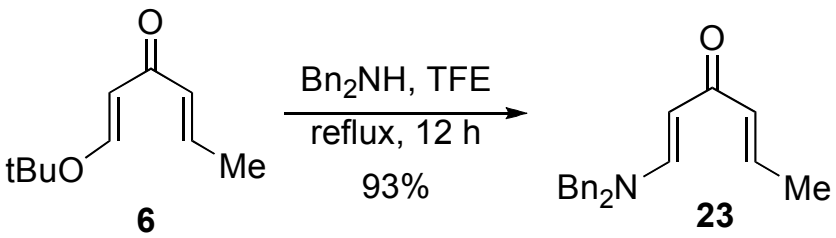

Scheme 4. Reaction of a dienone with a secondary amine. 
Table 1. Synthesis of dihydropyridones

\begin{tabular}{|c|c|c|c|c|c|}
\hline Entry & $\mathrm{R}$ & Amine & Product & $\mathbf{a}(\%)^{\mathrm{a}}$ & b (\%) $)^{a}$ \\
\hline 1 & $\mathrm{Me}$ & $\mathrm{Bn}-\mathrm{NH}_{2}$ & 7 & 92 & 5 \\
\hline 2 & $\mathrm{Me}$ & $\mathrm{H}_{2} \mathrm{~N} \leadsto$ & 11 & 87 & b \\
\hline 3 & $\mathrm{Me}$ & & 12 & 95 & b \\
\hline 4 & $\mathrm{Me}$ & & 13 & 94 & b \\
\hline 5 & $\mathrm{Me}$ & & 14 & 88 & b \\
\hline 6 & $\mathrm{Me}$ & $\mathrm{N}$ & 15 & 92 & b \\
\hline 7 & $\mathrm{Me}$ & & 16 & 91 & b \\
\hline 8 & $\mathrm{Me}$ & & 17 & 93 & b \\
\hline 9 & $\mathrm{Me}$ & & 18 & 92 & b \\
\hline 10 & $\mathrm{Me}$ & & 19 & 94 & b \\
\hline 11 & Et & $\mathrm{Bn}-\mathrm{NH}_{2}$ & 20 & 93 & 5 \\
\hline 12 & $-\mathrm{CH}_{2} \mathrm{CH}_{2}-\mathrm{t}-\mathrm{Bu}$ & $\mathrm{Bn}-\mathrm{NH}_{2}$ & 21 & 68 & 25 \\
\hline 13 & $\mathrm{Ph}$ & $\mathrm{Bn}-\mathrm{NH}_{2}$ & 22 & 12 & 85 \\
\hline
\end{tabular}

${ }^{\mathrm{a}}$ Isolated yield. ${ }^{\mathrm{b}}$ Only small amounts of $\mathbf{b}$ were formed.<smiles>[R]N/C=C\C(=O)/C=C\NC([R])=O</smiles> 
Table 2. Reaction of 6 with chiral amines

\begin{tabular}{|c|c|c|c|c|c|}
\hline Entry & Amine & Product & $\mathbf{a}(\%)^{a}$ & $\mathbf{b}(\%)^{\mathrm{a}}$ & c $(\%)^{a}$ \\
\hline 1 & & 30 & $83^{b}$ & 0 & 0 \\
\hline 2 & & 31 & $98^{c}$ & 0 & 0 \\
\hline 3 & & 32 & $92^{d}$ & 0 & 0 \\
\hline 4 & & 33 & 0 & 0 & 98 \\
\hline 5 & & 34 & $25^{\mathrm{e}}$ & 74 & 0 \\
\hline 6 & & 35 & $92^{\mathrm{f}}$ & 0 & 0 \\
\hline
\end{tabular}

${ }^{\mathrm{a}}$ Isolated yield. ${ }^{\mathrm{b}}$ Diastereomer ratio $(\mathrm{dr})$ by ${ }^{1} \mathrm{H}-\mathrm{NMR}=1: 1.5 .{ }^{\mathrm{c}} \mathrm{dr}=1: 1.7 .{ }^{\mathrm{d}} \mathrm{dr}=1: 1.1 .{ }^{\mathrm{e}} \mathrm{dr}=$ $1: 1{ }^{\mathrm{f}} \mathrm{dr}=1: 1$, but separable by chromatography.

Finally, we wondered whether the use of chiral amines would result in the formation of chiral dihydropyridones with either high diastereoselectivity or - if the diastereoselectivity were low - dihydropyridone diastereomers that could be easily separated. To that end, we studied the reaction of $\mathbf{6}$ with several chiral amines. The results are shown in Table 2. The use of amines 2428 resulted in the formation of dihydropyridones in good yields and, for 27, the formation of the vinylogous amide 33c. In cases where dihydropyridones were formed, they were not separable and diastereoselectivities were uniformly low. However, with amine 29, although there was essentially no diastereoselectivity, the products could be separated and the more polar product (35a') was found by X-ray crystallography to have the stereochemistry shown in Figure 1. 


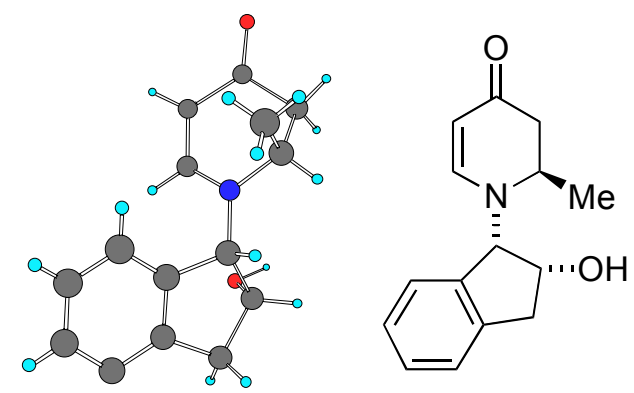

Figure 1. Structure of 35a'.

Hoping that 29 might produce dihydropyridone diastereomers that would be separable, regardless of the dienone, we examined a small number of dienones in the reaction with 29. These results are summarized in Table 3. Although only the structure of 35a-a' was established unequivocally by X-ray, we found a trend in the chemical shifts of the C-2 protons of the dihydropyridone diastereomers in this series that allowed us to make structural assignments. In general, the less polar dihydropyridone isomers have C-2- proton chemical shifts which are upfield relative to their more polar isomers. Table 3 shows that we had some success in separating the diastereomers of the dihydropyridones. However, for $\mathbf{4 1}$ simple chromatographic separation was not possible. Thus, while 29 appears to be a promising candidate for producing both enantiomeric series of selected dihydropyridones using this approach, it is not clear how effective it will be generally.

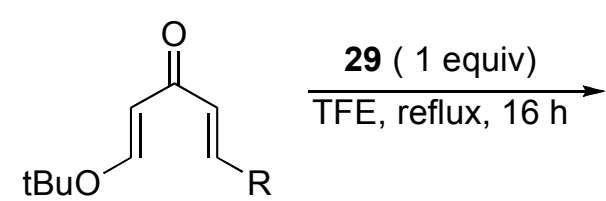

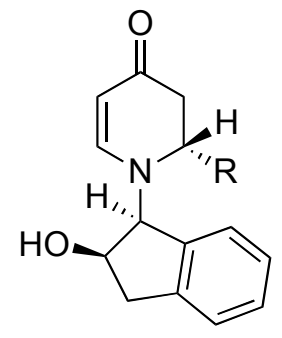

a, less polar

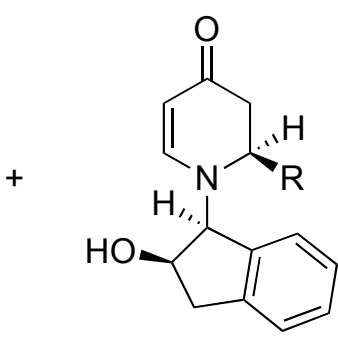

$\mathbf{a}^{\prime}$, more polar

Table 3. Reactions of dienones with amine $\mathbf{2 9}$

\begin{tabular}{cccccccc}
\hline Entry & $\mathrm{R}, \#$ & $\begin{array}{c}\mathrm{dr}^{\mathrm{a}} \\
\left(\mathbf{a}: \mathbf{a}^{\prime}\right)\end{array}$ & Product & $\mathbf{a}(\%)^{\mathrm{b}}$ & $\mathbf{a}^{\prime}(\%)^{\mathrm{b}}$ & $\begin{array}{c}\delta \mathrm{C} 2-\mathrm{Ha} \\
\mathrm{c}\end{array}$ & $\delta \mathrm{C} 2-\mathrm{Ha} \mathbf{a}^{\mathrm{c}}$ \\
\hline 1 & $\mathrm{Me}, \mathbf{6}$ & $1: 1$ & $\mathbf{3 5}$ & 47 & 45 & 3.67 & 3.82 \\
2 & $\mathrm{Et}, \mathbf{8}$ & $1: 1.3$ & $\mathbf{3 8}$ & 46 & 49 & 3.38 & 3.58 \\
3 & $\mathrm{n}-\mathrm{Bu}, \mathbf{3 6}$ & $1.5: 1$ & $\mathbf{3 9}$ & 63 & 35 & 3.41 & 3.64 \\
4 & $-\left(\mathrm{CH}_{2}\right)_{2} \mathrm{Bu} \mathrm{u}_{\mathrm{t}}, \mathbf{9}$ & $1: 1.3$ & $\mathbf{4 0}$ & 44 & 52 & 3.21 & 3.59 \\
5 & $-\left(\mathrm{CH}_{2}\right)_{2} \mathrm{Ph}, \mathbf{3 7}$ & $1: 1$ & $\mathbf{4 1}$ & $98^{\mathrm{d}}$ & $98^{\mathrm{d}}$ & 3.35 & 3.66 \\
\hline
\end{tabular}

${ }^{a}$ The dr was based on the crude ${ }^{1} \mathrm{H}$ - NMR. ${ }^{\mathrm{b}}$ Isolated yields. ${ }^{\mathrm{c}}$ In ppm; spectra taken in $\mathrm{CDCl}_{3}$.

${ }^{\mathrm{d}}$ The diastereomers were not separable, overall yield. 
In summary, we have demonstrated that dienones obtained from the retro-Nazarov reaction readily afford dihydropyridones upon reaction with primary amines. The chiral amine 29 is a promising candidate as an auxiliary that will allow access to both enantiomers of a given dihydropyridone. Studies concerning the refinement of such an auxiliary, other approaches to the enantioselective synthesis of dihydropyridones using this approach, and various applications are in progress and will be reported in due course.

\section{Experimental Section}

General Procedures. 2,2,2-Trifluoroethanol was distilled over anhydrous $\mathrm{CaSO}_{4}$ before use. Analytical TLC was performed on silica gel plates with F-254 indicator. Compounds were visualized by UV or development of the plate with staining by phosphomolybdic acid, potassium permanganate, or iodine followed by heating. Flash column chromatography was carried out on silica gel (40-63 $\mu \mathrm{m})$ with HPLC grade hexanes, ethyl acetate, and methanol. ${ }^{1} \mathrm{H}-\mathrm{NMR}$ spectra of $\mathrm{CDCl}_{3}$ solutions were recorded on Bruker AMX-250, 300 and $500 \mathrm{MHz}$ instruments, with tetramethylsilane $(0.005$ vol. $\%)$ as the internal reference. ${ }^{13} \mathrm{C}$ - NMR spectra were obtained on the same instruments at 62.9 or $75.5 \mathrm{MHz}$ with $\mathrm{CDCl}_{3}(\delta=77 \mathrm{ppm})$ as the internal reference. Chemical shifts are reported in ppm. Multiplicities are reported as $s$ (singlet), $b r$ (broad), $d$ (doublet), $t$ (triplet), $q$ (quartet), $m$ multiplet), $d d$ (doublet of doublets). In the ${ }^{1} \mathrm{H}$ - spectra of diastereomeric mixtures, the signals for the individual isomers are reported when possible.

High-resolution mass spectra were performed at the Department of Chemistry at Ohio State University, Columbus, $\mathrm{OH}$ and Old Dominion University, Norfolk, VA, by positive- ion electrospray, on a Bruker Apex-Qe FTICR-MS with an Apollo II source. X-ray diffraction data were obtained by Charles Barnes at the University of Missouri-Columbia, Columbia, MO. on a Bruker SMART CCD area detector on a three-circle goniometer with a molybdenum source and a graphite monochromator. Melting points were determined using a Fisher-Johns Hot Stage melting point apparatus and are uncorrected.

Synthesis of dihydropyridones. General procedure. A mixture of the 1,4-dienone-3-one (1.0 equiv.), amine (primary or secondary, 1.0 equiv.) and dried TFE $(c=0.04 M)$ were refluxed overnight (15-18 h). After reaction, evaporated the solvent and the resulting mixture was purified with flash column chromatography (ethyl acetate to 5-10\% MeOH-ethyl acetate) on silica gel.

1-Benzyl-2-methyl-2,3-dihydropyridin-4-(1H)-one (7a). Oily liquid; ${ }^{1} \mathrm{H}-\mathrm{NMR}(300 \mathrm{MHz}$, $\left.\mathrm{CDCl}_{3}\right), \delta$ 7.45-7.20 (m, 5H), 7.05 (d, $\left.J=7.45 \mathrm{~Hz}, 1 \mathrm{H}\right), 4.98(\mathrm{~d}, J=7.45 \mathrm{~Hz}, 1 \mathrm{H}), 4.39$ (q, 2H), $3.60(\mathrm{~m}, 1 \mathrm{H}), 2.71(\mathrm{dq}, J=16.3,6.60 \mathrm{~Hz}, 2 \mathrm{H}), 2.21(\mathrm{dd}, J=16.3,4.58 \mathrm{~Hz}, 1 \mathrm{H}), 1.23(\mathrm{~d}, J=6.6$ $\mathrm{Hz}, 3 \mathrm{H}) ;{ }^{13} \mathrm{C}-\mathrm{NMR}\left(75.5 \mathrm{MHz}, \mathrm{CDCl}_{3}\right) ; \delta 190.7,152.5,136.3,128.9,128.1,127.3,97.2,57.0$, 51.7, 42.4, 14.9; HRMS Calcd for $\mathrm{C}_{13} \mathrm{H}_{15} \mathrm{NONa}[\mathrm{M}+\mathrm{Na}]^{+} 224.1046$, Found 224.1043.

(1Z,4E)-1-(Benzylamino)hexa-1,4-dien-3-one (7b). Oily liquid; ${ }^{1} \mathrm{H}-\mathrm{NMR}(300.13 \mathrm{MHz}$, $\left.\mathrm{CDCl}_{3}\right), \delta 10.43(\mathrm{br}, 1 \mathrm{H}), 7.34-7.23(\mathrm{~m}, 5 \mathrm{H}), 6.85(\mathrm{dd}, J=12.59,7.36,1 \mathrm{H}), 6.73$ (ddd, $J=15.54$, $13.74,6.84 \mathrm{~Hz}, 1 \mathrm{H}), 6.08(\mathrm{dd}, J=13.74,1.60 \mathrm{~Hz}, 1 \mathrm{H}), 5.12(\mathrm{~d}, J=7.36,1 \mathrm{H}), 4.41(\mathrm{~d}, J=6.10$ 
$\mathrm{Hz}, 1 \mathrm{H}), 1.86(\mathrm{dd}, J=6.85,1.60 \mathrm{~Hz}, 1 \mathrm{H}) ;{ }^{13} \mathrm{C}-\mathrm{NMR}\left(75.5 \mathrm{MHz}, \mathrm{CDCl}_{3}\right), \delta 188.9,153.4,137.8$, 137.3, 132.3, 128.6, 127.8, 127.5, 127.0, 93.9, 52.5, 17.9; HRMS Calcd for $\mathrm{C}_{13} \mathrm{H}_{15} \mathrm{NONa}$ $[\mathrm{M}+\mathrm{Na}]^{+} 224.1046$, Found 224.1040.

1-Allyl-2-methyl-2,3-dihydropyridin-4(1H)-one (11a). Oily liquid, 87\%; ${ }^{1} \mathrm{H}-\mathrm{NMR}$ (300.13 $\left.\mathrm{MHz}, \mathrm{CDCl}_{3}\right), \delta 6.89(\mathrm{~d}, J=7.5 \mathrm{~Hz}, 1 \mathrm{H}), 5.81(\mathrm{~m}, 1 \mathrm{H}), 5.24(\mathrm{~m}, 2 \mathrm{H}), 4.89(\mathrm{~d}, J=7.5,1 \mathrm{H}), 3.77$ $(\mathrm{d}, J=5.8 \mathrm{~Hz}, 2 \mathrm{H}), 3.62(\mathrm{~m}, 1 \mathrm{H}), 2.70(\mathrm{dd}, \mathrm{J}=16.3,6.7 \mathrm{~Hz}, 1 \mathrm{H}), 2.18(\mathrm{dd}, J=16.3,4.6 \mathrm{~Hz}$, 1H), $1.19(\mathrm{~d}, J=6.7 \mathrm{~Hz}, 3 \mathrm{H}) ;{ }^{13} \mathrm{C}-\mathrm{NMR}\left(75.5 \mathrm{MHz}, \mathrm{CDCl}_{3}\right) ; \delta 190.7,152.0,133.1,118.5,97.1$, 55.6, 51.9, 42.4, 15.1; HRMS Calcd for $\mathrm{C}_{9} \mathrm{H}_{13} \mathrm{NONa}[\mathrm{M}+\mathrm{Na}]^{+}$174.0889, Found 174.0885.

2-Methyl-1-propyl-2,3-dihydropyridin-4(1H)-one (12a). Oily liquid, 95\%; ${ }^{1} \mathrm{H}-\mathrm{NMR}$ (300.13 $\left.\mathrm{MHz}, \mathrm{CDCl}_{3}\right), \delta 6.89(\mathrm{~d}, J=7.4 \mathrm{~Hz}, 1 \mathrm{H}), 4.87(\mathrm{~d}, J=7.4 \mathrm{~Hz}, 1 \mathrm{H}), 3.62(\mathrm{~m}, 1 \mathrm{H}), 3.22(\mathrm{~m}, 1 \mathrm{H})$, $3.05(\mathrm{~m}, 1 \mathrm{H}), 2.72(\mathrm{dd}, J=16.3,6.6 \mathrm{~Hz}, 1 \mathrm{H}), 2.19(\mathrm{dd}, J=16.3,4.3 \mathrm{~Hz}, 1 \mathrm{H}), 1.62(\mathrm{~m}, 2 \mathrm{H}), 1.21$ $(\mathrm{d}, J=6.6 \mathrm{~Hz}, 3 \mathrm{H}), 0.94(\mathrm{t}, J=7.4 \mathrm{~Hz}, 3 \mathrm{H}) ;{ }^{13} \mathrm{C}-\mathrm{NMR}\left(75.5 \mathrm{MHz}, \mathrm{CDCl}_{3}\right) ; \delta 190.4,152.4,96.3$, 55.0, 51.9, 42.3, 22.5, 15.2, 10.9; HRMS Calcd for $\mathrm{C}_{9} \mathrm{H}_{15} \mathrm{NONa}[\mathrm{M}+\mathrm{Na}]^{+} 176.1046$, Found 176.1040 .

1-(2-Hydroxyethyl)-2-methyl-2,3-dihydropyridin-4(1H)-one (13a). Oily liquid, 94\%; ${ }^{1} \mathrm{H}-$ NMR (250.13 MHz, $\left.\mathrm{CDCl}_{3}\right), \delta 7.01(\mathrm{~d}, J=7.3 \mathrm{~Hz}, 1 \mathrm{H}), 4.85(\mathrm{~d}, J=7.3 \mathrm{~Hz}, 1 \mathrm{H}), 4.36$ (br, 1H), $3.73(\mathrm{~m}, 3 \mathrm{H}), 3.33(\mathrm{~m}, 2 \mathrm{H}), 2.74(\mathrm{dd}, J=16.5,6.7 \mathrm{~Hz}, 1 \mathrm{H}), 2.16(\mathrm{dd}, J=16.5,4.7 \mathrm{~Hz}, 1 \mathrm{H}), 1.21$ $(\mathrm{d}, J=6.7 \mathrm{~Hz}, 3 \mathrm{H}) ;{ }^{13} \mathrm{C}-\mathrm{NMR}\left(62.9 \mathrm{MHz}, \mathrm{CDCl}_{3}\right) ; \delta 191.2,154.0,96.3,60.3,55.5,52.1,42.0$, 15.5; Anal. Calcd for $\mathrm{C}_{8} \mathrm{H}_{13} \mathrm{NO}_{2}$ : C, 61.91; H, 8.44. Found: C, 62.00; H, 8.54.

1-(2-Methoxyethyl)-2-methyl-2,3-dihydropyridin-4(1H)-one (14a). Oily liquid, 88\%; ${ }^{1} \mathrm{H}$ NMR (300.13 MHz, $\left.\mathrm{CDCl}_{3}\right), \delta 6.29(\mathrm{~d}, J=7.4 \mathrm{~Hz}, 1 \mathrm{H}), 4.88(\mathrm{~d}, J=7.4 \mathrm{~Hz}, 1 \mathrm{H}), 3.70(\mathrm{~m}, 1 \mathrm{H})$, 3.55-3.40 (m, 2H), 3.38-3.27 (m, $2 \mathrm{H}), 3.55(\mathrm{~s}, 3 \mathrm{H}), 2.73$ (dd. $J=16.2,6.6 \mathrm{~Hz}, 1 \mathrm{H}), 2.18(\mathrm{dd}, J=$ $16.2,4.4 \mathrm{~Hz}, 1 \mathrm{H}), 1.21(\mathrm{~d}, \mathrm{~J}=6.6 \mathrm{~Hz}, 3 \mathrm{H}) ;{ }^{13} \mathrm{C}-\mathrm{NMR}\left(75.5 \mathrm{MHz}, \mathrm{CDCl}_{3}\right) ; \delta 190.7,152.6,96.9$, 71.2, 59.0, 52.8, 52.6, 42.3, 15.3; Anal. Calcd for $\mathrm{C}_{9} \mathrm{H}_{15} \mathrm{NO}_{2}$ : C, 63.88; H, 8.93. Found: C, $63.89 ; \mathrm{H}, 8.53$.

1-(3-Hydroxypropyl)-2-methyl-2,3-dihydropyridin-4(1H)-one (15a). Oily liquid, 92\%; ${ }^{1} \mathrm{H}-$ NMR (300.13 MHz, $\left.\mathrm{CDCl}_{3}\right), \delta 6.98(\mathrm{~d}, J=7.3 \mathrm{~Hz}, 1 \mathrm{H}), 4.85(\mathrm{~d}, J=7.3 \mathrm{~Hz}, 1 \mathrm{H}), 3.80-3.60$ (m, 2H), 3.58 (br, 1H), 3.55 (t, $J=6.6 \mathrm{~Hz}, 2 \mathrm{H}), 2.69$ (dd, $J=16.4,6.7 \mathrm{~Hz}, 1 \mathrm{H}), 2.16$ (dd, $J=16.4$, $4.3 \mathrm{~Hz}, 1 \mathrm{H}), 1.80(\mathrm{~m}, 2 \mathrm{H}), 1.21(\mathrm{~d}, J=6.6 \mathrm{~Hz}, 3 \mathrm{H}) ;{ }^{13} \mathrm{C}-\mathrm{NMR}\left(75.5 \mathrm{MHz}, \mathrm{CDCl}_{3}\right) ; \delta 190.8$, 153.2, 96.7, 72.5, 69.6, 61.5, 52.8, 52.5, 42.2, 15.4; HRMS Calcd for $\mathrm{C}_{9} \mathrm{H}_{15} \mathrm{NO}_{2} \mathrm{Na}[\mathrm{M}+\mathrm{Na}]^{+}$ 192.0995, Found 192.0994.

1-(2-(2-Hydroxyethoxy)ethyl)-2-methyl-2,3-dihydropyridin-4(1H)-one (16a). Oily liquid, 91\%; ${ }^{1} \mathrm{H}-\mathrm{NMR}\left(300.13 \mathrm{MHz}, \mathrm{CDCl}_{3}\right), \delta 6.96(\mathrm{~d}, J=7.4 \mathrm{~Hz}, 1 \mathrm{H}), 4.87$ (d, J=7.4 Hz, 1H), 3.803.65 (m, 3H), 3.65-3.52 (m, 4H), 3.40-3.25 (m, 2H), 2.90 (br., 1H), 2.71 (dd, $J=16.3,6.6 \mathrm{~Hz}$, $1 \mathrm{H}), 2.16(\mathrm{dd}, J=16.3,4.6 \mathrm{~Hz}, 1 \mathrm{H}), 1.20(\mathrm{~d}, J=6.7 \mathrm{~Hz}, 3 \mathrm{H}) ;{ }^{13} \mathrm{C}-\mathrm{NMR}\left(75.5 \mathrm{MHz}, \mathrm{CDCl}_{3}\right) ; \delta$ $190.8,153.2,96.7,72.5,69.6,61.5,52.8$, 52.5, 42.2, 15.4; HRMS Calcd for $\mathrm{C}_{10} \mathrm{H}_{17} \mathrm{NO}_{3} \mathrm{Na}$ $[\mathrm{M}+\mathrm{Na}]^{+} 222.1101$, Found 222.1093.

2-Methyl-1-(pyridin-2-ylmethyl)-2,3-dihydropyridin-4(1H)-one (17a). Oily liquid, 93\%; ${ }^{1} \mathrm{H}$ NMR (250.13 MHz, $\left.\mathrm{CDCl}_{3}\right), \delta 8.58(\mathrm{dd}, J=3.9,0.6 \mathrm{~Hz}, 1 \mathrm{H}), 7.72(\mathrm{dt}, J=7.7,1.8 \mathrm{~Hz}, 1 \mathrm{H}), 7.25$ (m, 2H), 7.08 (d, $J=7.4 \mathrm{~Hz}, 1 \mathrm{H}), 4.98$ (d, $J=7.4 \mathrm{~Hz}, 1 \mathrm{H}), 4.47$ (dd, $\left.-\mathrm{CH}_{2}-\mathrm{Ar}, 2 \mathrm{H}\right), 3.64$ (hex, $J$ 
$=16.3,5.4 \mathrm{~Hz}, 1 \mathrm{H}), 1.21(\mathrm{~d}, J=6.7 \mathrm{~Hz}, 3 \mathrm{H}) ;{ }^{13} \mathrm{C}-\mathrm{NMR}\left(62.9 \mathrm{MHz}, \mathrm{CDCl}_{3}\right) ; \delta 190.9,156.6$, 153.0, 149.8, 137.0, 122.9, 121.3, 97.8, 58.7, 52.3, 42.6, 15.4; Anal. Calcd for $\mathrm{C}_{12} \mathrm{H}_{14} \mathrm{~N}_{2} \mathrm{O}$ : C, 71.26; H, 6.98. Found: C, 71.12; H, 7.12.

1-(2-Bromophenylethyl)-2-methyl-2,3-dihydropyridin-4(1H)-one (18a). Oily liquid, 92\%; ${ }^{1} \mathrm{H}-$ NMR (300.13 MHz, $\left.\mathrm{CDCl}_{3}\right), \delta 7.56(\mathrm{dd}, J=7.9,1.2 \mathrm{~Hz}, 1 \mathrm{H}), 7.23(\mathrm{dq}, J=5.9,1.2 \mathrm{~Hz}, 2 \mathrm{H})$, $7.13(\mathrm{dt}, J=7.9,2.0 \mathrm{~Hz}, 1 \mathrm{H}), 6.80(\mathrm{~d}, J=7.5 \mathrm{~Hz}, 1 \mathrm{H}), 4.86(\mathrm{dd}, J=7.5,0.63 \mathrm{~Hz}, 1 \mathrm{H}), 3.60(\mathrm{~m}$, $1 \mathrm{H}), 3.44(\mathrm{~m}, 2 \mathrm{H}), 3.0(\mathrm{~m}, 2 \mathrm{H}), 2.67(\mathrm{dd}, J=16.3,6.6 \mathrm{~Hz}, 1 \mathrm{H}), 2.18$ (ddd, $J=16.3,4.6,0.63 \mathrm{~Hz}$, $1 \mathrm{H}), 1.24(\mathrm{~d}, J=6.6 \mathrm{~Hz}, 3 \mathrm{H}) ;{ }^{13} \mathrm{C}-\mathrm{NMR}\left(75.5 \mathrm{MHz}, \mathrm{CDCl}_{3}\right) ; \delta 190.6,152.0,136.9,133.0,131.2$, 128.7, 127.7, 124.2, 97.1, 52.7, 52.4, 42.4, 36.6, 15.5; Anal. Calcd for $\mathrm{C}_{14} \mathrm{H}_{16} \mathrm{BrNO}$ : C, 57.16; $\mathrm{H}$, 5.48. Found: C, 57.09; H, 5.36.

1-(2-(1H-Indol-3-yl)ethyl)-2-methyl-2,3-dihydropyridin-4(1H)-one (19a). Oily liquid, 94\%; ${ }^{1} \mathrm{H}-\mathrm{NMR}\left(250.13 \mathrm{MHz}, \mathrm{CDCl}_{3}\right.$ ), $\delta 9.03$ (br., $\left.1 \mathrm{H}\right), 7.56$ (d, $\left.J=7.6 \mathrm{~Hz}, 1 \mathrm{H}\right), 7.39$ (d, $J=7.8 \mathrm{~Hz}$, $1 \mathrm{H}), 7.3-7.1(\mathrm{~m}, 2 \mathrm{H}), 7.0(\mathrm{~d}, J=2.3 \mathrm{~Hz}, 1 \mathrm{H}), 6.69(\mathrm{~d}, J=7.3 \mathrm{~Hz}, 1 \mathrm{H}), 4.8(\mathrm{~d}, J=7.3 \mathrm{~Hz}, 1 \mathrm{H})$, 3.65-3.35 (m, 3H), 3.1-2.9 (m, 2H), $2.65(\mathrm{dd}, J=16.3,6.7 \mathrm{~Hz}, 1 \mathrm{H}), 2.16(\mathrm{dd}, J=16.3,4.6 \mathrm{~Hz}$, 1H), $1.22(\mathrm{~d}, J=6.7 \mathrm{~Hz}, 3 \mathrm{H}) ;{ }^{13} \mathrm{C}-\mathrm{NMR}\left(62.9 \mathrm{MHz}, \mathrm{CDCl}_{3}\right) ; \delta 190.7,152.8,136.4,126.7,122.9$, 122.0, 119.3, 118.0, 111.6, 111.1, 96.0, 53.6, 52.2, 42.2, 25.9, 15.5; Anal. Calcd for $\mathrm{C}_{16} \mathrm{H}_{18} \mathrm{~N}_{2} \mathrm{O}$ : C, 75.56; H, 7.13. Found: C, 75.36; H, 7.03.

1-Benzyl-2-ethyl-2,3-dihydropyridin-4(1H)-one (20a). Oily liquid, 93\%; ${ }^{1} \mathrm{H}-\mathrm{NMR}$ (250.13 $\left.\mathrm{MHz}, \mathrm{CDCl}_{3}\right), \delta$ 7.5-7.2 (m, 5H), $7.05(\mathrm{~d}, J=7.5,1 \mathrm{H}), 4.94(\mathrm{~d}, J=7.5 \mathrm{~Hz}, 1 \mathrm{H}), 4.45\left(\mathrm{q},-\boldsymbol{C H}_{2^{-}}\right.$ $\mathrm{Ph}, 2 \mathrm{H}), 3.34$ (m 1H), 2.68 (dd, $J=16.0,6.8 \mathrm{~Hz}, 1 \mathrm{H}), 2.34$ (dd, $J=16.0,2.96 \mathrm{~Hz}, 1 \mathrm{H}), 1.9-1.6$ $(\mathrm{m}, 2 \mathrm{H}), 0.91(\mathrm{t}, J=7.5 \mathrm{~Hz}, 3 \mathrm{H}) ;{ }^{13} \mathrm{C}-\mathrm{NMR}\left(62.9 \mathrm{MHz}, \mathrm{CDCl}_{3}\right) ; \delta 190.6,152.6,136.5,128.9$, 128.1, 127.2, 97.1, 57.7, 57.4, 38.7, 21.4; HRMS Calcd for $\mathrm{C}_{14} \mathrm{H}_{17} \mathrm{NONa}[\mathrm{M}+\mathrm{Na}]^{+}$238.1202, Found 238.1199.

1-Benzyl-2-(3,3-dimethylbutyl)-2,3-dihydropyridin-4(1H)-one (21a). Oily liquid, 68\%; ${ }^{1} \mathrm{H}-$ NMR (300.13 MHz, $\left.\mathrm{CDCl}_{3}\right), \delta$ 7.5-7.2 (m, 5H), $7.05(\mathrm{~d}, J=7.40 \mathrm{~Hz}, 1 \mathrm{H}), 4.93(\mathrm{~d}, J=7.40 \mathrm{~Hz}$, 1H), 4.39 (q, - $\left.\boldsymbol{C H}_{2}-\mathrm{Ph}, 1 \mathrm{H}\right), 3.34(\mathrm{~m}, 1 \mathrm{H}), 2.67$ (dd, $\left.J=16.4,6.8 \mathrm{~Hz}, 1 \mathrm{H}\right), 2.25$ (dd, $J=16.4$, $3.42 \mathrm{~Hz}, 1 \mathrm{H}), 1.8-1.5(\mathrm{~m}, 2 \mathrm{H}), 1.24(\mathrm{dt}, J=12.6,5.16 \mathrm{~Hz}, 1 \mathrm{H}), 1.02(\mathrm{dt}, J=12.6,4.5 \mathrm{~Hz}, 1 \mathrm{H})$, 0.85 (s, 9H); ${ }^{13} \mathrm{C}-\mathrm{NMR}\left(75.5 \mathrm{MHz}, \mathrm{CDCl}_{3}\right) ; \delta 190.5,152.5,136.5,128.9,128.1,127.3,97.1$, 57.8, 56.8, 39.4, 39.3, 30.1, 29.1, 23.4; Anal. Calcd for $\mathrm{C}_{18} \mathrm{H}_{25} \mathrm{NO}$ : C, 79.66; H, 9.28. Found: C, 79.82; H, 9.15 .

1-Benzyl-2-phenyl-2,3-dihydropyridin-4(1H)-one (22a). Oily liquid, 12\%; ${ }^{1} \mathrm{H}-\mathrm{NMR}(250.13$ $\mathrm{MHz}, \mathrm{CDCl}_{3}$ ), $\delta 7.4-7.23(\mathrm{~m}, 10 \mathrm{H}), 5.09$ (d, $\left.J=7.64 \mathrm{~Hz}, 1 \mathrm{H}\right), 4.51(\mathrm{t}, J=7.63 \mathrm{~Hz}, 1 \mathrm{H}), 4.35$ (d, $J=15.08 \mathrm{~Hz}, 1 \mathrm{H}), 4.13(\mathrm{~d}, J=15.08 \mathrm{~Hz}, 1 \mathrm{H}), 2.77(\mathrm{dq}, J=16.0,7.07 \mathrm{~Hz}, 2 \mathrm{H}) ;{ }^{13} \mathrm{C}-\mathrm{NMR}(75.5$ $\left.\mathrm{MHz}, \mathrm{CDCl}_{3}\right) ; \delta 190.3,154.0,138.5,128.9,128.8,128.2,128.1,127.6,127.0,98.7,60.6,57.1$, 43.6; Anal. Calcd for $\mathrm{C}_{18} \mathrm{H}_{17} \mathrm{NO}$ : C, 82.10; H, 6.51. Found: C, 82.00; H, 6.41.

(1Z,4E)-1-(Benzylamino)-5-phenylpenta-1,4-dien-3-one (22b). Oily liquid, 85\%; 1H-NMR (250.13 MHz, CDCl3), $\delta 10.6$ (br., $1 \mathrm{H}), 7.5-7.4$ (m, 3H), 7.35-7.25 (m, 8H), 6.94 (dd, $J=12.6$, $7.3 \mathrm{~Hz}, 1 \mathrm{H}), 6.72(\mathrm{~d}, J=15.6 \mathrm{~Hz}, 1 \mathrm{H}), 5.29$ (d, $J=7.3,1 \mathrm{H}), 4.46(\mathrm{~d}, J=6.08 \mathrm{~Hz}, 1 \mathrm{H}), 13 \mathrm{C}-$ NMR (75.5 MHz, $\left.\mathrm{CDCl}_{3}\right), \delta$ 188.2, 153.8, 138.2, 137.6, 135.6, 129.2, 128.7, 128.6, 127.9, 127.7, 
127.6, 127.1, 95.2, 52.6; Anal. Calcd for $\mathrm{C}_{18} \mathrm{H}_{17} \mathrm{NO}$ : C, 82.10; H, 6.51. Found: C, 81.99; H, 6.32 .

(1E,4E)-1-(Dibenzylamino)hexa-1,4-dien-3-one (23). Oily liquid, 93\%; ${ }^{1} \mathrm{H}-\mathrm{NMR}$ (300.13 $\left.\mathrm{MHz}, \mathrm{CDCl}_{3}\right), \delta 8.02(\mathrm{~d}, J=12.7 \mathrm{~Hz}, 1 \mathrm{H}), 7.40-7.25(\mathrm{~m}, 6 \mathrm{H}), 7.22-7.10(\mathrm{~m}, 4 \mathrm{H}), 6.78(\mathrm{ddd}, J=$ $15.8,6.8,1.6 \mathrm{~Hz}, 1 \mathrm{H}), 5.42(\mathrm{~d}, J=12.7 \mathrm{~Hz}, 1 \mathrm{H}), 4.35(\mathrm{~s}, 4 \mathrm{H}), 1.84(\mathrm{dd}, J=6.8,1.6 \mathrm{~Hz}, 3 \mathrm{H})$; ${ }^{13} \mathrm{C}-\mathrm{NMR}\left(75.5 \mathrm{MHz}, \mathrm{CDCl}_{3}\right) ; \delta 187.5,152.9,138.0,135.4,132.6,128.7,127.4,96.0,17.9$; HRMS Calcd for $\mathrm{C}_{20} \mathrm{H}_{21} \mathrm{NONa}[\mathrm{M}+\mathrm{Na}]^{+} 314.1515$, Found 314.1517.

1-((1S,2R)-1-Hydroxy-1-phenylpropan-2-yl)-2-methyl-2,3-dihydropyridin-4(1H)-one (30a). Oily liquid, 83\% (dr = 1:1.5); ${ }^{1} \mathrm{H}-\mathrm{NMR}\left(300.13 \mathrm{MHz}, \mathrm{CDCl}_{3}\right), \delta$ 7.4-7.2 (m, 10H), $7.06(\mathrm{~d}, J=$ $6.7 \mathrm{~Hz}, 1 \mathrm{H}), 6.95(\mathrm{~d}, J=7.5 \mathrm{~Hz}, 1 \mathrm{H}), 4.87-4.77(\mathrm{~m}, 3 \mathrm{H}), 4.65(\mathrm{~d}, J=6.7 \mathrm{~Hz}, 1 \mathrm{H}), 3.72-3.60$ (m, $1 \mathrm{H}), 3.59-3.46(\mathrm{~m}, 1 \mathrm{H}), 3.45-3.27(\mathrm{~m}, 2 \mathrm{H}), 2.63(\mathrm{dd}, J=16.4,6.7 \mathrm{~Hz}, 1 \mathrm{H}), 2.39$ (dd, $J=16.4$, $7.2 \mathrm{~Hz}, 1 \mathrm{H}), 2.22-1.86(\mathrm{~m}, 2 \mathrm{H}), 1.31(\mathrm{~d}, J=6.7 \mathrm{~Hz}, 3 \mathrm{H}), 1.29(\mathrm{~d}, J=6.7 \mathrm{~Hz}, 3 \mathrm{H}), 1.14(\mathrm{~d}, J=$ $6.7 \mathrm{~Hz}, 3 \mathrm{H}), 1.13(\mathrm{~d}, J=6.7 \mathrm{~Hz}, 3 \mathrm{H}) ;{ }^{13} \mathrm{C}-\mathrm{NMR}\left(75.5 \mathrm{MHz}, \mathrm{CDCl}_{3}\right) ; \delta 191.3,190.7,151.9$, $148.7,141.7,141.4,128.3,128.2,127.8,127.7,126.4,126.1,96.1,95.6,76.7,76.4,65.1,63.0$, 55.1, 50.5, 41.8, 41.1, 16.6, 15.3, 15.2, 13.7; Anal. Calcd for $\mathrm{C}_{15} \mathrm{H}_{19} \mathrm{NO}_{2}$ : C, 73.44; H, 7.81. Found: C, 73.18; H, 7.77.

1-((R)-2-Hydroxy-1-phenylethyl)-2-methyl-2,3-dihydropyridin-4(1H)-one (31a). Oily liquid, $98 \%(\mathrm{dr}=1: 1.7) ;{ }^{1} \mathrm{H}-\mathrm{NMR}\left(250.13 \mathrm{MHz}, \mathrm{CDCl}_{3}\right), \delta 7.45(\mathrm{~d}, J=7.6 \mathrm{~Hz}, 1 \mathrm{H}), 7.40-7.20(\mathrm{~m}$, $10 \mathrm{H}), 7.03(\mathrm{~d}, J=7.3,1 \mathrm{H}), 5.01(\mathrm{~d}, J=7.6 \mathrm{~Hz}, 1 \mathrm{H}), 4.89$ (d, $J=7.3 \mathrm{~Hz}, 1 \mathrm{H}), 4.55-4.42(\mathrm{~m}, 2 \mathrm{H})$, 4.20-3.90 (m, 4H), $3.85(\mathrm{dt}, J=6.7,2.5 \mathrm{~Hz}, 1 \mathrm{H}), 3.50(\mathrm{dt}, J=6.7,2.9 \mathrm{~Hz}, 1 \mathrm{H}), 2.82(\mathrm{dd}, J=$ $16.4,6.8 \mathrm{~Hz}, 1 \mathrm{H}), 2.71(\mathrm{dd}, J=16.4,6.8 \mathrm{~Hz}, 1 \mathrm{H}), 2.14(\mathrm{dd}, J=8.3,2.3 \mathrm{~Hz}, 1 \mathrm{H}), 2.08(\mathrm{dd}, J$ $=8.3,2.3 \mathrm{~Hz}, 1 \mathrm{H}), 1.25(\mathrm{~d}, J=6.7 \mathrm{~Hz}, 3 \mathrm{H}), 1.13(\mathrm{~d}, J=6.7 \mathrm{~Hz}, 3 \mathrm{H}) ;{ }^{13} \mathrm{C}-\mathrm{NMR}(62.9 \mathrm{MHz}$, $\left.\mathrm{CDCl}_{3}\right) ; \delta 191.2,190.9,150.6,150.0,137.6,136.9,129.0,128.4,127.6,126.9,96.6,96.2,67.3$, 63.1, 63.0, 53.7, 52.0, 42.0, 41.8, 16.2, 15.0; HRMS Calcd for $\mathrm{C}_{14} \mathrm{H}_{17} \mathrm{NO}_{2} \mathrm{Na}[\mathrm{M}+\mathrm{Na}]^{+} 254.1152$, Found 254.1154.

2-Methyl-1-((R)-1-phenylethyl)-2,3-dihydropyridin-4(1H)-one (32a). Oily liquid, 92\% ( dr = 1:1.1); ${ }^{1} \mathrm{H}-\mathrm{NMR}\left(250.13 \mathrm{MHz}, \mathrm{CDCl}_{3}\right), \delta$ 7.45-7.2(m, $\left.12 \mathrm{H}\right), 6.92(\mathrm{dd}, \mathrm{J}=7.5,0.9 \mathrm{~Hz}, 1 \mathrm{H}), 5.00$ $(\mathrm{dd}, \mathrm{J}=7.5,0.9 \mathrm{~Hz}, 1 \mathrm{H}), 4.88(\mathrm{dd}, \mathrm{J}=7.5,0.9 \mathrm{~Hz}, 1 \mathrm{H}), 4.54(\mathrm{q}, \mathrm{J}=6.9 \mathrm{~Hz}, 1 \mathrm{H}), 4.46(\mathrm{q}, \mathrm{J}=6.9$ $\mathrm{Hz}, 1 \mathrm{H}), 3.71(\mathrm{~m}, 1 \mathrm{H}), 3.50(\mathrm{~m}, 1 \mathrm{H}), 2.67(\mathrm{ddd}, \mathrm{J}=14.8,6.9,1.4 \mathrm{~Hz}, 2 \mathrm{H}), 2.07$ (ddd, J = 9.2, 3.2, $1.0 \mathrm{~Hz}, 1 \mathrm{H}), 2.13(\mathrm{ddd}, \mathrm{J}=10.0,4.1,0.9 \mathrm{~Hz}, 1 \mathrm{H}), 1.63(\mathrm{~d}, \mathrm{~J}=6.9 \mathrm{~Hz}, 3 \mathrm{H}), 1.58(\mathrm{~d}, \mathrm{~J}=6.9$, $3 \mathrm{H}), 1.24(\mathrm{~d}, \mathrm{~J}=6.7 \mathrm{~Hz}, 3 \mathrm{H}), 1.17(\mathrm{~d}, \mathrm{~J}=6.7 \mathrm{~Hz}, 3 \mathrm{H}) ;{ }^{13} \mathrm{C}-\mathrm{NMR}\left(62.9 \mathrm{MHz}, \mathrm{CDCl}_{3}\right) ; \delta 190.6$, $190.5,149.7,147.4,142.3,140.3,128.9,128.8,128.1,127.9,126.9,125.8,96.9,96.5,61.2$, 60.3, 52.2, 51.4, 42.4, 41.9, 21.5, 19.5, 16.4, 15.1. Anal. Calcd for $\mathrm{C}_{14} \mathrm{H}_{17} \mathrm{NO}$ : C, 78.10; H, 7.96. Found: C, 77.88; H, 8.08.

\section{(1E,4E)-1-(Benzyl((1S,2S)-1-hydroxy-1-phenylpropan-2-yl)amino)hexa-1,4-dien-3-one}

(33c). Oily liquid, 98\%; ${ }^{1} \mathrm{H}-\mathrm{NMR}\left(500.13 \mathrm{MHz}, \mathrm{CDCl}_{3}\right), \delta 7.87(\mathrm{~d}, J=12.4 \mathrm{~Hz}, 1 \mathrm{H}), 7.4-7.2(\mathrm{~m}$, $5 \mathrm{H}), 6.72$ (dddd, $J=15.4,6.8 \mathrm{~Hz}, 1 \mathrm{H}), 6.13$ (dd, $J=15.4,1.6 \mathrm{~Hz}, 1 \mathrm{H}), 4.80$ (br., 1H), 4.56 (d, $J$ $=8.4 \mathrm{~Hz}, 1 \mathrm{H}), 3.65(\mathrm{~m}, 1 \mathrm{H}), 2.82(\mathrm{~s}, 3 \mathrm{H}), 1.83(\mathrm{dd}, J=6.8,1.6 \mathrm{~Hz}, 1 \mathrm{H}), 0.99(\mathrm{~d}, J=6.8 \mathrm{~Hz}$, $3 \mathrm{H}) ;{ }^{13} \mathrm{C}-\mathrm{NMR}\left(62.9 \mathrm{MHz}, \mathrm{CDCl}_{3}\right) ; \delta 187.1,153.9,141.5,137.5,132.6,128.4,127.8,126.9$, 
95.0, 75.8, 68.0(br), 32.0(br), 18.0, 15.6; Anal. Calcd for $\mathrm{C}_{16} \mathrm{H}_{21} \mathrm{NO}_{2}$ : C, 74.10; H, 8.16. Found: C, 74.30; H, 7.97 .

1-((1S,2S)-2-Hydroxy-6-nitro-2,3-dihydro-1H-inden-1-yl)-2-methyl-2,3-dihydropyridin4(1H)-one (34a). Oily liquid, 25\% (dr = 1: 1.05); ${ }^{1} \mathrm{H}-\mathrm{NMR}\left(300.13 \mathrm{MHz}, \mathrm{CDCl}_{3}\right), \delta 8.20(\mathrm{t}, J=$ $2.4 \mathrm{~Hz}, 1 \mathrm{H}), 8.17(\mathrm{t}, J=2.4 \mathrm{~Hz}, 1 \mathrm{H}), 8.04(\mathrm{~s}, 1 \mathrm{H}), 7.96(\mathrm{~s}, 1 \mathrm{H}), 7.42(\mathrm{~d}, J=8.3 \mathrm{~Hz}, 1 \mathrm{H}), 7.40$ (d, $J=8.3 \mathrm{~Hz}, 1 \mathrm{H}), 6.95(\mathrm{~d}, J=7.5 \mathrm{~Hz}, 1 \mathrm{H}), 6.85(\mathrm{~d}, J=7.5 \mathrm{~Hz}, 1 \mathrm{H}), 4.99$ (t, $J=7.2 \mathrm{~Hz}, 2 \mathrm{H}), 4.75$ $(\mathrm{t}, J=6.4 \mathrm{~Hz}, 2 \mathrm{H}), 4.67(\mathrm{q}, J=7.4 \mathrm{~Hz}, 1 \mathrm{H}), 4.58(\mathrm{q}, J=7.8 \mathrm{~Hz}, 1 \mathrm{H}), 3.99-3.90(\mathrm{~m}, 1 \mathrm{H}), 3.80-$ $3.78(\mathrm{~m}, 1 \mathrm{H}), 6.79(\mathrm{dt}, J=17.4,7.5 \mathrm{~Hz}, 2 \mathrm{H}), 3.1-2.8(\mathrm{~m}, 4 \mathrm{H}), 2.35-2.20(\mathrm{~m}, 2 \mathrm{H}), 1.45(\mathrm{~d}, J=6.7$ $\mathrm{Hz}, 3 \mathrm{H}), 1.38(\mathrm{~d}, J=6.7 \mathrm{~Hz}, 3 \mathrm{H}) ;{ }^{13} \mathrm{C}-\mathrm{NMR}\left(75.5 \mathrm{MHz}, \mathrm{CDCl}_{3}\right) ; \delta 191.9,191.7,150.4,149.2$, $147.9,147.8,147.5,147.3,140.5,140.1,126.4,126.2,124.5,124.4,119.7,119.1,98.8,98.4$, 79.7, 78.4, 75.2, 73.6, 53.8, 53.6, 42.7, 42.3, 38.7, 38.3, 17.0, 16.9; Anal. Calcd for $\mathrm{C}_{15} \mathrm{H}_{16} \mathrm{~N}_{2} \mathrm{O}_{4}$ : C, 62.49; H, 5.59. Found: C, 62.32; H, 5.69.

(1Z,4E)-1-((1S,2S)-2-Hydroxy-6-nitro-2,3-dihydro-1 $H$-inden-1-ylamino)hexa-1,4-dien-3-one (34b). Oily liquid, 74\% ( $\mathrm{dr}=1: 1.05) ;{ }^{1} \mathrm{H}-\mathrm{NMR}$ (300.13 MHz, $\mathrm{CDCl}_{3}$ ), $\delta 10.25$ (br., $\left.1 \mathrm{H}\right), 8.13$ (dd, $J=8.2,2.1 \mathrm{~Hz}, 1 \mathrm{H}), 8.04(\mathrm{~s}, 1 \mathrm{H}), 7.32$ (d, $J=8.2 \mathrm{~Hz}, 1 \mathrm{H}), 6.93$ (br., 1H), 6.66 (dddd, $J=$ 15.5, 1.6 Hz, 1H), 6.04 (dd, $J=15.5,1.6 \mathrm{~Hz}, 1 \mathrm{H}), 5.13$ (d, $J=7.4 \mathrm{~Hz}, 1 \mathrm{H}), 4.48$ (br., 1H), 4.42 $9 \mathrm{q}, J=7.5 \mathrm{~Hz}, 1 \mathrm{H}), 3.25(\mathrm{dd}, J=16.5,7.3 \mathrm{~Hz}, 1 \mathrm{H}), 2.92(\mathrm{dd}, J=16.5,8.7 \mathrm{~Hz}, 1 \mathrm{H}), 1.87(\mathrm{dd}, J=$ 6.8, 1.6 Hz, 3H); ${ }^{13} \mathrm{C}-\mathrm{NMR}\left(75.5 \mathrm{MHz}, \mathrm{CDCl}_{3}\right) ; \delta 189.7,153.1,147.7,146.5,141.6,138.4$, 132.2 , 125.7, 124.1, 119.3, 94.8, 80.7, 70.9, 37.6, 18.1; Anal. Calcd for $\mathrm{C}_{15} \mathrm{H}_{16} \mathrm{~N}_{2} \mathrm{O}_{4}$ : C, 62.49; $\mathrm{H}$, 5.59. Found: C, 62.36; H, 5.40.

(S)-1-((1S,2R)-2-Hydroxy-2,3-dihydro-1H-inden-1-yl)-2-methyl-2,3-dihydropyridin-4(1H)one (35a). Oily liquid, 47\%; ${ }^{1} \mathrm{H}-\mathrm{NMR}\left(250.13 \mathrm{MHz}, \mathrm{CDCl}_{3}\right), \delta 7.30-7.20(\mathrm{~m}, 4 \mathrm{H}), 7.02$ (dd, $J=$ 7.4, $0.6 \mathrm{~Hz}, 1 \mathrm{H}), 4.85$ (dd, $J=7.4,0.5 \mathrm{~Hz}, 1 \mathrm{H}), 4.68$ (s, 3H), 3.66 (dt, $J=6.7,2.3 \mathrm{~Hz}, 1 \mathrm{H}), 3.16$ (m, 1H), 2.99 (d, $J=16.3 \mathrm{~Hz}, 1 \mathrm{H}), 2.96$ (d, $J=16.3 \mathrm{~Hz}, 1 \mathrm{H}), 2.12$ (ddd, $J=16.3,3.4,0.8 \mathrm{~Hz}$, 1H), $1.20(\mathrm{~d}, J=6.7 \mathrm{~Hz}, 3 \mathrm{H}) ;{ }^{13} \mathrm{C}-\mathrm{NMR}\left(62.9 \mathrm{MHz}, \mathrm{CDCl}_{3}\right) ; \delta 191.6,153.1,140.6,138.5,128.7$, 127.0, 125.5, 125.2, 96.2, 73.4, 71.3, 54.2, 42.2, 42.1, 40.0, 16.6; HRMS Calcd for $\mathrm{C}_{15} \mathrm{H}_{17} \mathrm{NO}_{2} \mathrm{Na}[\mathrm{M}+\mathrm{Na}]^{+}$266.1152, Found 266.1148.

(R)-1-((1S,2R)-2-Hydroxy-2,3-dihydro-1H-inden-1-yl)-2-methyl-2,3-dihydropyridin-4(1H)one (35a'). White solid, mp 167-169 ${ }^{\circ} \mathrm{C}, 45 \%$; ${ }^{1} \mathrm{H}-\mathrm{NMR}\left(250.13 \mathrm{MHz}, \mathrm{CDCl}_{3}\right), \delta$ 7.30-7.20 (m, $3 \mathrm{H}), 7.15-7.05$ (m, 1H), 6.79 (d, $J=7.6 \mathrm{~Hz}, 1 \mathrm{H}), 5.13$ (br., $1 \mathrm{H}), 4.75$ (d, $J=7.6 \mathrm{~Hz}, 1 \mathrm{H}), 4.64$ (m, 2H), $3.82(\mathrm{~m}, 1 \mathrm{H}), 3.10-3.98(\mathrm{~m}, 2 \mathrm{H}), 2.90(\mathrm{dd}, J=16.5,7.0 \mathrm{~Hz}, 1 \mathrm{H}), 2.23(\mathrm{dd}, J=16.5,3.8$ $\mathrm{Hz}, 1 \mathrm{H}), 1.39$ (d, J = 7.0 Hz, 3H); ${ }^{13} \mathrm{C}-\mathrm{NMR}\left(62.9 \mathrm{MHz}, \mathrm{CDCl}_{3}\right) ; \delta 191.6,151.7,141.3,138.1$, 128.6, 127.2, 125.6, 124.5, 96.5, 72.6, 69.6, 54.4, 41.8, 39.2, 16.3; Anal. Calcd for $\mathrm{C}_{15} \mathrm{H}_{17} \mathrm{NO}_{2}$ : C, 74.05; H, 7.04. Found: C, 74.13; H, 6.95.

(S)-2-Ethyl-1-((1S,2R)-2-hydroxy-2,3-dihydro-1H-inden-1-yl)-2,3-dihydropyridin-4(1H)one (38a). Oily liquid, 46\%; ${ }^{1} \mathrm{H}-\mathrm{NMR}\left(250.13 \mathrm{MHz}, \mathrm{CDCl}_{3}\right), \delta 7.30-7.20(\mathrm{~m}, 4 \mathrm{H}), 7.05(\mathrm{~d}, \mathrm{~J}=$ $7.4 \mathrm{~Hz}, 1 \mathrm{H}), 4.86(\mathrm{~d}, \mathrm{~J}=7.4 \mathrm{~Hz}, 1 \mathrm{H}), 4.70(\mathrm{~m}, 2 \mathrm{H}), 4.14$ (s, br., 1H), $3.86(\mathrm{~m}, 1 \mathrm{H}), 3.19$ (dd, J = 16.4, 5.2 Hz, 1H), $3.1-2.9$ (m, 2H), 2.38 (d, J = 16.4 Hz, 1H), 1.96 (m, 1H), 1.48 (m, 1H), 1.26 $(\mathrm{s}, 1 \mathrm{H}), 0.80(\mathrm{t}, \mathrm{J}=7.43 \mathrm{~Hz}, 3 \mathrm{H}) ;{ }^{13} \mathrm{C}-\mathrm{NMR}\left(62.9 \mathrm{MHz}, \mathrm{CDCl}_{3}\right) ; \delta 191.5,153.4,140.5,138.7$, 
128.8, 127.1, 125.5, 125.1, 96.6, 73.9, 71.6, 60.5, 40.0, 38.0, 22.5, 10.0; HRMS Calcd for $\mathrm{C}_{16} \mathrm{H}_{19} \mathrm{NO}_{2} \mathrm{Na}[\mathrm{M}+\mathrm{Na}]^{+} 280.1308$, Found 280.1309.

(R)-2-Ethyl-1-((1S,2R)-2-hydroxy-2,3-dihydro-1H-inden-1-yl)-2,3-dihydropyridin-4(1H)one (38a'). Oily liquid, 49\%; ${ }^{1} \mathrm{H}-\mathrm{NMR}\left(250.13 \mathrm{MHz}, \mathrm{CDCl}_{3}\right), \delta$ 7.30-7.20 (m, 3H), 7.20 - 7.10 $(\mathrm{m}, 1 \mathrm{H}), 6.83(\mathrm{~d}, J=7.5, \mathrm{~Hz}, 1 \mathrm{H}), 5.29(\mathrm{~m}, 2 \mathrm{H}), 4.68(\mathrm{~m}, 3 \mathrm{H}), 3.58(\mathrm{~m}, 1 \mathrm{H}), 3.2-3.0(\mathrm{~m}, 2 \mathrm{H})$, $3.0-2.8(\mathrm{~m}, 1 \mathrm{H}), 2.37(\mathrm{dd}, J=16.7,2.7 \mathrm{~Hz}, 1 \mathrm{H}), 1.88(\mathrm{~m}, 2 \mathrm{H}), 1.01(\mathrm{t}, J=7.5 \mathrm{~Hz}, 3 \mathrm{H}) ;{ }^{13} \mathrm{C}-$ NMR $\left(62.9 \mathrm{MHz}, \mathrm{CDCl}_{3}\right) ; \delta 191.9,151.9,141.4,137.9,128.7,127.1,125.8,124.4,96.7,72.8$, 70.7, 60.1, 39.1, 38.6, 23.0, 10.0; HRMS Calcd for $\mathrm{C}_{16} \mathrm{H}_{19} \mathrm{NO}_{2} \mathrm{Na}[\mathrm{M}+\mathrm{Na}]^{+} 280.1308$, Found 280.1310 .

(S)-2-Butyl-1-((1S,2R)-2-hydroxy-2,3-dihydro-1H-inden-1-yl)-2,3-dihydropyridin-4(1H)one (39a). Oily liquid, 63\%; ${ }^{1} \mathrm{H}-\mathrm{NMR}\left(250.13 \mathrm{MHz}, \mathrm{CDCl}_{3}\right), \delta$ 7.30-7.25 (m, 4H), $7.04(\mathrm{~d}, J=$ 7.4, Hz, 1H), 4.89 (dd, $J=7.4, \mathrm{~Hz}, 1 \mathrm{H}), 4.70(\mathrm{~s}, 2 \mathrm{H}), 3.65(\mathrm{~m}, 1 \mathrm{H}), 3.41(\mathrm{~m}, 1 \mathrm{H}), 3.25-3.15(\mathrm{~m}$, $2 \mathrm{H}), 3.10-2.90(\mathrm{~m}, 2 \mathrm{H}), 2.36(\mathrm{~d}, J=15.9 \mathrm{~Hz}, 1 \mathrm{H}), 2.0(\mathrm{~m}, 1 \mathrm{H}), 1.5-1.1(\mathrm{~m}, 4 \mathrm{H}), 1.1-0.9(\mathrm{~m}$, 2H), $0.81(\mathrm{t}, J=7.0 \mathrm{~Hz}, 3 \mathrm{H}) ;{ }^{13} \mathrm{C}-\mathrm{NMR}\left(62.9 \mathrm{MHz}, \mathrm{CDCl}_{3}\right) ; \delta 191.5,153.0,140.4,138.4,128.9$, 128.2, 127.2, 125.5, 125.4, 96.8, 73.8, 71.6, 58.6, 39.9, 38.6, 29.1, 27.5, 22.3, 13.8; HRMS Calcd for $\mathrm{C}_{18} \mathrm{H}_{23} \mathrm{NO}_{2} \mathrm{Na}[\mathrm{M}+\mathrm{Na}]^{+}$308.1621, Found 308.1626.

(R)-2-Butyl-1-((1S,2R)-2-hydroxy-2,3-dihydro-1H-inden-1-yl)-2,3-dihydropyridin-4(1H)one (39a'). Oily liquid, 35\%; ${ }^{1} \mathrm{H}-\mathrm{NMR}\left(250.13 \mathrm{MHz}, \mathrm{CDCl}_{3}\right), \delta$ 7.32-7.20 (m, 3H), $7.18-7.10$ (m, 1H), 6.83 (d, $J=7.4, \mathrm{~Hz}, 1 \mathrm{H}), 4.75$ (d, $J=7.4, \mathrm{~Hz}, 1 \mathrm{H}), 4.71-4.60$ (m, 2H), 4.54 (br., 1H), $3.64(\mathrm{~m}, 1 \mathrm{H}), 3.20-3.05(\mathrm{~m}, 2 \mathrm{H}), 3.05-2.85(\mathrm{~m}, 1 \mathrm{H}), 2.37(\mathrm{dd}, J=17.0,2.1 \mathrm{~Hz}, 1 \mathrm{H}), 1.9-1.7$ $(\mathrm{m}, 2 \mathrm{H}), 1.45-1.25(\mathrm{~m}, 6 \mathrm{H}), 1.0-0.9(\mathrm{~m}, 4 \mathrm{H}) ;{ }^{13} \mathrm{C}-\mathrm{NMR}\left(62.9 \mathrm{MHz}, \mathrm{CDCl}_{3}\right) ; \delta 191.7,151.4$, $141.3,137.8,128.7,127.2,125.8,124.5,96.9,73.0,70.7,58.9,39.1,29.6,27.7,22.6,13.9$; HRMS Calcd for $\mathrm{C}_{18} \mathrm{H}_{23} \mathrm{NO}_{2} \mathrm{Na}[\mathrm{M}+\mathrm{Na}]^{+} 308.1621$, Found 308.1626.

(S)-2-(3,3-Dimethylbutyl)-1-((1S,2R)-2-hydroxy-2,3-dihydro-1H-inden-1-yl)-2,3-

dihydropyridin-4(1H)-one (40a). Oily liquid, 44\%; ${ }^{1} \mathrm{H}-\mathrm{NMR}\left(250.13 \mathrm{MHz}, \mathrm{CDCl}_{3}\right), \delta$ 7.337.25 (m, 4H), 7.1 (d, $J=7.40 \mathrm{~Hz}, 1 \mathrm{H}), 4.91$ (d, $J=7.4, \mathrm{~Hz}, 1 \mathrm{H}), 4.69$ (s, 2H), 3.97 (s, br., 1H), $3.30-3.20(\mathrm{~m}, 2 \mathrm{H}), 3.10-2.80(\mathrm{~m}, 2 \mathrm{H}), 2.30(\mathrm{~d}, J=8.2 \mathrm{~Hz}, 1 \mathrm{H}), 2.0-1.85(\mathrm{~m}, 1 \mathrm{H}), 1.26-1.10$ $(\mathrm{m}, 3 \mathrm{H}), 0.76(\mathrm{~s}, 9 \mathrm{H}) ;{ }^{13} \mathrm{C}-\mathrm{NMR}\left(62.9 \mathrm{MHz}, \mathrm{CDCl}_{3}\right) ; \delta 191.7,153.3,140.7,138.0,129.1,127.2$, 125.8, 125.5, 96.8, 73.6, 71.4, 58.5, 39.7, 39.6, 38.5, 30.1, 29.1, 24.7; HRMS Calcd for $\mathrm{C}_{20} \mathrm{H}_{27} \mathrm{NO}_{2} \mathrm{Na}[\mathrm{M}+\mathrm{Na}]^{+}$336.1934, Found 336.1936.

(R)-2-(3,3-Dimethylbutyl)-1-((1S,2R)-2-hydroxy-2,3-dihydro-1 H-inden-1-yl)-2,3-

dihydropyridin-4(1H)-one (40a'). Oily liquid, 52\%; ${ }^{1} \mathrm{H}-\mathrm{NMR}\left(250.13 \mathrm{MHz}, \mathrm{CDCl}_{3}\right), \delta 7.32-$ $7.20(\mathrm{~m}, 3 \mathrm{H}), 7.20-7.10(\mathrm{~m}, 1 \mathrm{H}), 6.84(\mathrm{~d}, J=7.40 \mathrm{~Hz}, 1 \mathrm{H}), 5.20$ (s, br., 1H), 4.69 (m, 2H), 3.59 (m, 1H), $3.20-3.10(\mathrm{~m}, 2 \mathrm{H}), 3.10-2.85(\mathrm{dd}, J=16.8,7.30 \mathrm{~Hz}, 1 \mathrm{H}), 2.35(\mathrm{dd}, J=16.8,2.1 \mathrm{~Hz}$, $1 \mathrm{H}), 3.61(\mathrm{~m}, 2 \mathrm{H}), 1.41-1.10(\mathrm{~m}, 3 \mathrm{H}), 0.89(\mathrm{~s}, 9 \mathrm{H}) ;{ }^{13} \mathrm{C}-\mathrm{NMR}\left(62.9 \mathrm{MHz}, \mathrm{CDCl}_{3}\right) ; \delta 191.9$, $151.8,141.4,137.9,128.7,127.2,125.8,124.4,96.8,72.9,71.1,59.5,39.6,39.3,39.2,30.2$, 29.2, 25.2; HRMS Calcd for $\mathrm{C}_{20} \mathrm{H}_{27} \mathrm{NO}_{2} \mathrm{Na}[\mathrm{M}+\mathrm{Na}]^{+} 336.1934$, Found 336.1937.

1-((1S,2R)-2-Hydroxy-2,3-dihydro-1H-inden-1-yl)-2-phenethyl-2,3-dihydropyridin-4(1H)one (41a, 41a'). Oily liquid, 98\% ( $\mathrm{dr}=1: 1)$; ${ }^{1} \mathrm{H}-\mathrm{NMR}\left(250.13 \mathrm{MHz}, \mathrm{CDCl}_{3}\right), \delta$ 7.35-7.10 (m, $18 \mathrm{H}), 6.93$ (d, $J=7.30 \mathrm{~Hz}, 1 \mathrm{H}), 6.84$ (d, $J=7.50 \mathrm{~Hz}, 1 \mathrm{H}), 5.06$ (s, br., $1 \mathrm{H}), 4.89$ (d, $J=7.30 \mathrm{~Hz}$, 
1H), 2H), 4.80 (d, J = 7.50 Hz, 1H), $4.64(\mathrm{~m}, 5 \mathrm{H}), 4.30$ (s, br., 1H), 3.37 (m, 1H), $3.20-2.80$ $(\mathrm{m}, 7 \mathrm{H}), 2.80-2.70(\mathrm{~m}, 2 \mathrm{H}), 2.70-2.50(\mathrm{~m}, 2 \mathrm{H}), 2.49-2.20(\mathrm{~m}, 6 \mathrm{H}), 2.20-2.00(\mathrm{~m}, 2 \mathrm{H}), 1.60$ $(\mathrm{m}, 1 \mathrm{H}) ;{ }^{13} \mathrm{C}-\mathrm{NMR}\left(62.9 \mathrm{MHz}, \mathrm{CDCl}_{3}\right) ; \delta 191.6,153.7,151.7,141.4,140.7,140.6,140.4$, $138.0,137.8,129.1,128.8,128.6,128.4,128.2$, 128.1, 127.3, 127.2, 126.2, 125.9, 125.8, 125.7, 125.6, 124.5, 96.6, 73.2, 72.9, 71.6, 70.7, 58.2, 56.7, 39.8, 39.1, 38.7, 37.9, 31.6, 31.3, 30.6 ; HRMS Calcd for $\mathrm{C}_{22} \mathrm{H}_{23} \mathrm{NO}_{2} \mathrm{Na}[\mathrm{M}+\mathrm{Na}]^{+} 356.1621$, Found 356.1622.

\section{Acknowledgements}

This work was supported by the National Science Foundation to whom we are grateful. We thank Dr. Leland O. Weigel (Lilly) for a gift of 28. We gratefully acknowledge donations of fluorinated solvents from Halocarbon Products Corp. We thank Dr. Charles L. Barnes for acquisition of X-ray data.

\section{References}

1. (a) Comins, D. L. J. Heterocycl. Chem. 1999, 36, 1491. (b) Joseph, S.; Comins, D. L. Curr. Opin. Drug. Discov. Devel. 2002, 5, 870.

2. (a) Harmata, M.; Lee Dong, R. J. Am. Chem. Soc. 2002, 124, 14328. (b) Harmata, M.; Schreiner, P. R.; Lee, D. R.; Kirchhoefer, P. L. J. Am. Chem. Soc. 2004, 126, 10954. (c) Harmata, M.; Lee, D. R.; Barnes, C. L. Org. Lett. 2005, 7, 1881.

3. Dong, D.; Bi, X.; Liu, Q.; Cong, F. Chem. Commun. 2005, 3580.

4. The coupling constant between the C-5.C-6 protons was $7.4 \mathrm{~Hz}$ for 7a. The coupling constants for the $\mathrm{C}-1 / \mathrm{C}-2$ and $\mathrm{C}-4 / \mathrm{C}-5$ protons for $7 \mathbf{b}$ were $7.4 \mathrm{~Hz}$, and $13.7 \mathrm{~Hz}$, respectively. The $\mathrm{C}-1 / \mathrm{C}-2$ alkene group of $\mathbf{7 b}$ was thus assigned the $Z$ - stereochemistry. 